Supplementary materials for

Microbial roles in dissolved organic matter transformation in full-scale wastewater treatment processes revealed by reactomics and comparative genomics

Liye Wang, Yuan Lin, Lin Ye, Yuli Qian, Yufei Shi, Ke Xu, Hongqiang Ren, Jinju Geng*

State Key Laboratory of Pollution Control and Resource Reuse, School of the Environment, Nanjing University, Nanjing 210023, Jiangsu, PR China

*Corresponding author: Professor Jinju Geng; Address: School of the Environment, Nanjing University, N.O.163, Xianlin Avenue, Nanjing 210023, Jiangsu, PR China; Email: jjgeng@nju.edu.cn; Phone:+862589680360

Total pages: 24

Texts: 6

Tables: 2

Figures: 11 


\section{Supplementary Texts}

Text S1 3D-EEM analysis

Three-dimensional excitation-emission matrix (3D-EEM) fluorescence spectroscopy was obtained using a luminescence spectrometry RF-6000 (Shimadzu). The scanning wavelength range was set as: excitation wavelength (Ex) 240-550 nm, emission wavelength (Em) 280-550 nm, $1 \mathrm{~nm}$ increments. The bandpass for the excitation and emission wavelength was $5 \mathrm{~nm}$, and the scan speed was $2400 \mathrm{~nm} / \mathrm{min}$. The water Raman peak of ultrapure water was considered as a reference. All EEMs were corrected by blanks (ultrapure water), and the calibration process was conducted by FL Solutions (v4.0). Parallel factor (PARAFAC) analysis was performed using DOMFluor ${ }^{1}$ (v1.7) on MATLAB (vR2020b, MathWorks, USA) to separate fluorescence spectra from the 3D-EEM fluorescence spectra. 


\section{Text S2 Definitions of BIX, HIX, and FIX}

Biological index (BIX), indicative the extent of autochthonous contribution, was derived by dividing the emission intensity at a wavelength of $380 \mathrm{~nm}$ to the maximum emission intensity at wavelengths between 420 and $435 \mathrm{~nm}$, and obtained at an excitation wavelength of $310 \mathrm{~nm}$,

$$
B I X=\frac{\text { Fluo }_{310 / 380}}{\max \left(\text { Fluo }_{310 / 420 \rightarrow 435)}\right.}
$$

Humification index (HIX) reflects the extent of humification of DOM. It is quantified by dividing the integrated area of fluorescence intensity under emission spectra 435480 by the area in the region $300-345 \mathrm{~nm}$, under an excitation wavelength of $254 \mathrm{~nm}$,

$$
H I X=\frac{\operatorname{area}_{254 / 435 \rightarrow 480}}{\text { area }_{254 / 300 \rightarrow 345}}
$$

Fluorescence index (FIX) is related to the relative contribution of DOM from microbial sources or plant degradation sources. It is quantified as the ratio of fluorescence intensities between 470 and $520 \mathrm{~nm}$ under the excitation wavelength of $370 \mathrm{~nm}$,

$$
F I X=\frac{\text { Fluo }_{370 / 470}}{\text { Fluo }_{370 / 520}}
$$


Text S3 Attributes of DOM molecules and samples

(1) Double bond equivalent (DBE) $)^{2}$ :

$$
D B E=\frac{2 \times C+N+P-H+2}{2}
$$

DBE indicates the unsaturation of a specific molecule.

(2) Nominal oxidation state of carbon $(\mathrm{NOSC})^{3}$ :

$$
N O S C=4-\frac{4 \times C+H-2 \times O-3 \times N-2 \times S+5 \times P}{C}
$$

(3) Modified aromaticity index $\left(\mathrm{AI}_{\mathrm{mod}}\right)^{4}$ :

$$
A I_{\text {mod }}=\frac{1+C-0.5 \times O-S-0.5 \times(N+P+H)}{C-0.5 \times O-N-S-P}
$$

where in (1)(2)(3), C, H, O, N, S and P are the number of carbon, hydrogen, oxygen, nitrogen, sulfur, and phosphorus atoms in a given molecular formula

(4) Molecular lability index (MLBL) ${ }^{5}$ :

$$
M L B_{L}=\frac{N_{H: C>1.5}}{N_{t}}
$$

where $\mathrm{N}_{\mathrm{H}: \mathrm{C}>1.5}$ is the number of formulae with $\mathrm{H}: \mathrm{C}$ ratio $>1.5$, while $\mathrm{N}_{\mathrm{t}}$ is the total number of formulae in that sample. Commonly, molecules with higher $\mathrm{H}: \mathrm{C}$ ratio are more bio-liable.

(5) Chemodiversity (Gini-Simpson index ${ }^{6}$ ):

$$
D_{A}=1-\sum_{i=1}^{N} p_{i}^{2}
$$

where $\mathrm{p}_{\mathrm{i}}$ is the relative intensity of the molecular formula $\mathrm{i}$.

(6) Functional molecular diversity:

$$
D_{F}(c)=\sum_{i=1}^{N-1} \sum_{j=i+1}^{N} p_{i} \cdot p_{j} \cdot\left|c_{i}-c_{j}\right|
$$

where $\mathrm{c}_{\mathrm{i}}$ is a quantitative chemical property of the molecular formula i (calculated based on the molecular weight, $\mathrm{H}: \mathrm{C}, \mathrm{N}: \mathrm{C}, \mathrm{DBE}, \mathrm{NOSC}$, and $\mathrm{AI}_{\mathrm{mod}}$ ). For all molecule pairs (i, $j$ ) in the sample, the absolute difference in this property $c_{i}-c_{j}$ is weighted by the sumnormalized signal intensities $\mathrm{p}_{\mathrm{i}}$ and $\mathrm{p}_{\mathrm{j}}$ of the two molecules. The value $\mathrm{D}_{\mathrm{F}}(\mathrm{c})$ can be interpreted as the expected difference between two molecules with respect to the chosen property. $^{7}$ 
Text S4 Categories of DOM molecules

Based on $\mathrm{H}: \mathrm{C}, \mathrm{O}: \mathrm{C}, \mathrm{N}: \mathrm{C}$ ratios and modified aromatic index $\left(\mathrm{AI}_{\mathrm{mod}}\right)$, molecules can be divided into eight compound categories:

(1) Nitrogen-rich (proteins-like) compounds ${ }^{3,8}$ :

$1.5 \leq \mathrm{H} / \mathrm{C} \leq 2.2,0.2 \leq \mathrm{O} / \mathrm{C} \leq 0.52,0.178 \leq \mathrm{N} / \mathrm{C} \leq 0.44$, and $\mathrm{P} / \mathrm{C} \leq 0.06$

(2) Amino sugars-like compounds ${ }^{3}$ :

$1.5 \leq \mathrm{H} / \mathrm{C} \leq 2.2,0.52 \leq \mathrm{O} / \mathrm{C} \leq 0.7,0.07<\mathrm{N} / \mathrm{C} \leq 0.182$, and $\mathrm{P} / \mathrm{C}<0.167$

(3) Aliphatic compounds ${ }^{4,9}$ :

$$
1.5 \leq \mathrm{H} / \mathrm{C} \leq 2.2 \text {, and not (1) or (2) }
$$

(4) Highly unsaturated and phenolic compounds ${ }^{4}$ :

$$
0.7 \leq \mathrm{H} / \mathrm{C}<1.5,0 \leq \mathrm{O} / \mathrm{C}<0.1 \text {, and } \mathrm{AI}_{\text {mod }} \leq 0.5
$$

(5) Polyphenols 4 :

$$
\mathrm{H} / \mathrm{C}<1.5 \text {, and } 0.5<\mathrm{AImod}<0.67
$$

(6) Condensed aromatics ${ }^{4}$ :

$$
0.3 \leq \mathrm{H} / \mathrm{C} \leq 1.25 \text {, and } \mathrm{AImod} \geq 0.67
$$

(7) Lignin-like compounds ${ }^{3,8}$ :

$$
0.6 \leq \mathrm{H} / \mathrm{C}<1.5,0.1 \leq \mathrm{O} / \mathrm{C}<0.67 \text {, and } \mathrm{AI}_{\text {mod }}<0.67
$$

(8) Tannins-like compounds ${ }^{3,8}$ :

$$
0.5 \leq \mathrm{H} / \mathrm{C}<1.5,0.6 \leq \mathrm{O} / \mathrm{C} \leq 1 \text {, and } \mathrm{AI}_{\bmod }<0.67
$$


Text S5 Metagenomic sequencing

All extraction processes were performed under sterile conditions. DNA was extracted using FastDNA ${ }^{\circledR}$ SPIN Kit for Soil (MP Biomedicals, USA) following the manufacturer's protocol. DNA extracts were stored at $-80{ }^{\circ} \mathrm{C}$ until shipment on dry ice to Novogene Co., LTD (Beijing, China).

DNA was quantified using the Qubit dsDNA High Sensitivity assay and, when quantity permitted, DNA quality was assessed using agarose gel electrophoresis. Sample DNA, $\sim 200 \mathrm{ng}$, was sheared to $\sim 500 \mathrm{bp}$ using a Covaris LE220 focused ultrasonicator. The sheared DNA fragments were size selected by double-solid phase reversible immobilization and then selected fragments were end-repaired, A-tailed and ligated with Illumina-compatible adaptors (Integrated DNA Technologies) containing a unique molecular index barcode for each sample library. The prepared libraries were quantified using KAPA Biosystem's next-generation sequencing library qPCR kit and run on a Roche LightCycler 480 real-time PCR instrument. $\sim 100$ pM DNA for each sample was sequenced on a NovaSeq 6000 platform, following a $2 \times 150$ indexed run recipe, with a read depth of $\sim 6 \mathrm{Gbp}$ per sample. Detailed processes are based on ref $^{10}$. 
Text S6 Metagenomic data processing

\section{Quality control}

Quality control of raw reads was performed using Trimmomatic ${ }^{11}$ (v0.39). Adapters were removed from the paired read using reference "TruSeq3-PE.fa" with seed mismatches $<2$, palindrome clip threshold $>40$, simple clip threshold $>15$, and minAdapterLength $>8 \mathrm{bp}$. A sliding window trimming was performed with window size $=4$, required quality $>15$, and minimal length $>50 \mathrm{bp}$.

\section{Assembly}

Qualified reads from A/A/O samples and A/O samples were co-assembled separately by MEGAHIT ${ }^{12}$ (v1.2.9) with default kmer list and parameter "--min-contig-len 1000".

\section{Binning}

Binning was performed using MaxBin213 (v2.2.6) with parameters "min_contig_length 1000 -max_iteration 55", Metabat214 (v2.13) with parameters "-m 1500", and CONCOCT ${ }^{15}$ (v1.1.0) with default parameters. MetaWRAP ${ }^{16}$ (v1.3) was used to dereplicate and aggregate the similar bins from these bin sets with parameters "-c 50 -x 10". Potential contaminative contigs were identified using RefineM's (v0.1.2). ${ }^{17}$ Outliers (contigs had GC or tetranucleotide distance outside the $98^{\text {th }}$ percentile of the expected distributions or conflicting phylum-level taxonomy with specific MAGs) were then removed from the corresponding MAGs. The quality of retrieved MAGs was estimated by checkM ${ }^{18}$ (v1.0.13). Putative tRNA genes and rRNA genes in each MAG were retrieved using tRNAscan-SE ${ }^{19}$ (v2.0.6) and Infernal ${ }^{20}$ (v1.1.3). Only MAGs met with the high-quality (completeness $>90 \%$, contamination $<5 \%$, presence of the $23 \mathrm{~S}, 16 \mathrm{~S}, 5 \mathrm{~S}$ rRNA genes, and at least 18 tRNAs) or mediumquality (completeness $\geq 50 \%$ and contamination $<10 \%$ ) criteria of the MIMAG standard ${ }^{21}$ were included in the downstream analysis. Abovementioned calculationintensity processes were performed on High Performance Computing Center, Nanjing University.

\section{Taxonomic classification}

MAGs were taxonomically classified using GTDB-Tk ${ }^{22}$ (v1.02). The corresponding NCBI taxonomy was retrieved based on the genome metadata file provided by GTDB (https://data.ace.uq.edu.au/public/gtdb/data/releases/release95/95.0/auxillary_files/gtd b_vs_ncbi_r95_bacteria.xlsx) and manually verified. 


\section{Functional annotation}

Functional annotation was conducted according to $\mathrm{He}$ et al. ${ }^{23}$. Specifically, METABOLIC $^{24}$ (v.4.0) was used to search predicted open reading frames (ORFs) against a curated set of KEGG, TIGRfam, Pfam, and custom HMM profiles. For specific sets of proteins that are often misannotated due to high sequence similarity despite divergent function (for example, amo $A B C / p m o A B C$ ), an additional motifvalidation step was performed. On the basis of the presence/absence and abundances of this manually curated set of marker genes, the presence/absence and abundances of metabolic capacities encoded by each genome were determined. 


\section{Supplementary Tables}

Table S1 Recovery of DOM

\begin{tabular}{cccc}
\hline Sample & Coverage $(\%)$ & Sample & Coverage (\%) \\
\hline Sep-Influent & 52.3 & Jan-Influent & 51.4 \\
Sep-AGC & 56.7 & Jan-AGC & 54.1 \\
Sep-AAO-Pre & 89.1 & Jan-AAO-Pre & 83.5 \\
Sep-AAO-A1 & 94.5 & Jan-AAO-A1 & 93.1 \\
Sep-AAO-A2 & 95.1 & Jan-AAO-A2 & 94.0 \\
Sep-AAO-O & 93.6 & Jan-AAO-O & 92.7 \\
Sep-AAO-Sed & 90.2 & Jan-AAO-Sed & 95.4 \\
Sep-AO-A & 67.5 & Jan-AO-A & 74.2 \\
Sep-AO-O & 90.9 & Jan-AO-O & 92.8 \\
Sep-AO-Sed & 95.3 & Jan-AO-Sed & 89.2 \\
Sep-MIX & 94.4 & Jan-MIX & 95.7 \\
Sep-Effluent & 93.9 & Jan-Effluent & 94.0 \\
\hline
\end{tabular}


Table S2 Statistics of the pairwise PERMANOVA testing

\begin{tabular}{ccccc}
\hline Paired groups & $\begin{array}{c}\text { Sums of } \\
\text { squares }\end{array}$ & F Model & $\begin{array}{c}\text { Variation } \\
(\mathrm{R} 2)\end{array}$ & $P$-value \\
\hline AAO/AO & 0.118 & 1.28 & 0.084 & 0.23 \\
Influent/AAO & 0.937 & 5.21 & 0.303 & 0.003 \\
AAO/Effluent & 0.042 & 0.41 & 0.033 & 0.73 \\
Influent/AO & 0.799 & 3.95 & 0.330 & 0.01 \\
AO/Effluent & 0.100 & 1.17 & 0.127 & 0.286 \\
Influent/Effluent & 0.723 & 2.79 & 0.318 & 0.029 \\
Influent/Others & 1.092 & 7.95 & 0.266 & 0.001 \\
Effluent/Others & 0.115 & 0.64 & 0.028 & 0.67 \\
Sep/Jan & 0.806 & 5.36 & 0.196 & 0.0009 \\
\hline
\end{tabular}

Influent: Influent and AGC from both Sep-2019 and Jan-2020.

AAO: AAO-Pre, AAO-A1, AAO-A2, AAO-O, AAO-Sed from both Sep-2019 and Jan-2020.

AO: AO-A, AO-O, AO-Sed from both Sep-2019 and Jan-2020.

Effluent: MIX and Effluent from both Sep-2019 and Jan-2020.

Sep: All samples from Sep-2019.

Jan: All samples from Jan-2020.

Permutations $=999$ 


\section{Supplementary Figures}

a
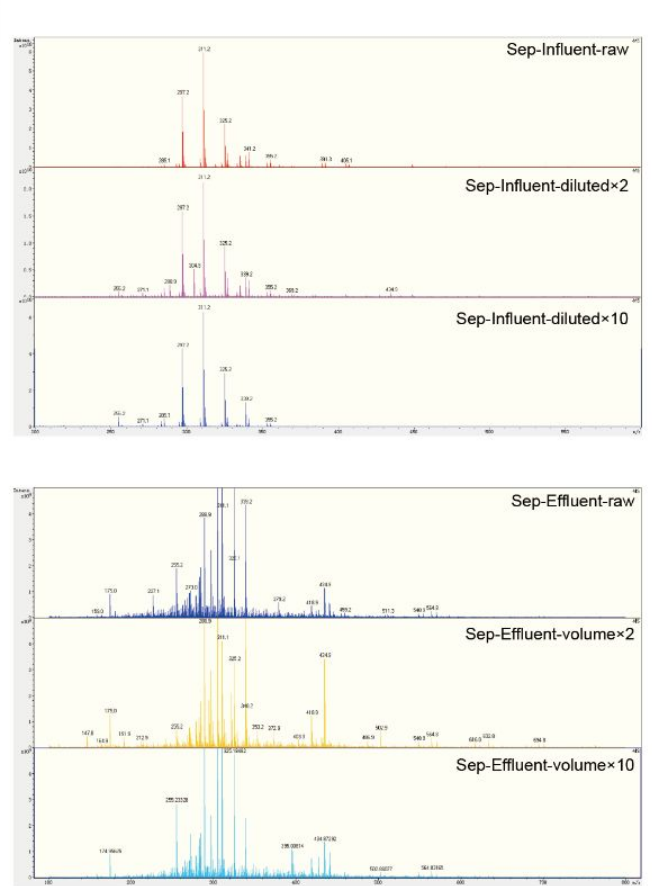

$\mathrm{b}$

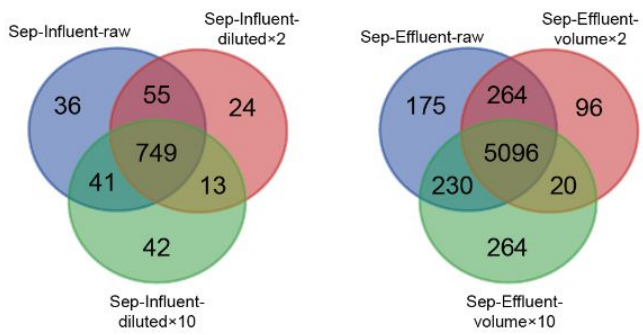

C

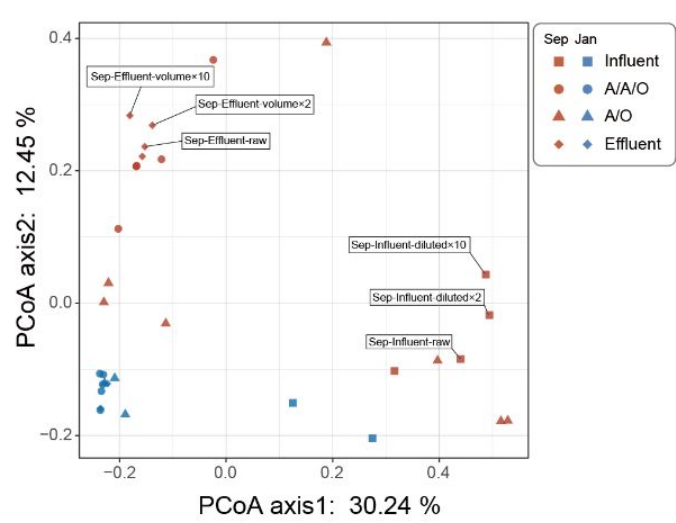

Figure S1 Recovery rates experiments. To evaluate the effect of low recovery on the composition of identified DOM, we tested the DOM compositions of Sep-Influent with different dilution levels (1 L raw, 2-times diluted, and 10-time diluted samples) and Sep-Effluent with different filter volumes (1L, 2L and 10L raw samples). The recoveries of diluted Sep-Influent samples increased $(52.3 \%, 68.2 \%, 82.1 \%)$ while the recoveries of higher volumes of Sep-Effluent decreased (93.9\%, 73.4\%, 49.8\%). (a) Raw spectra of samples. (b) The overlap of identified formulae in samples. (c) PCoA analysis exhibits the distances between test samples in the context of all samples. 

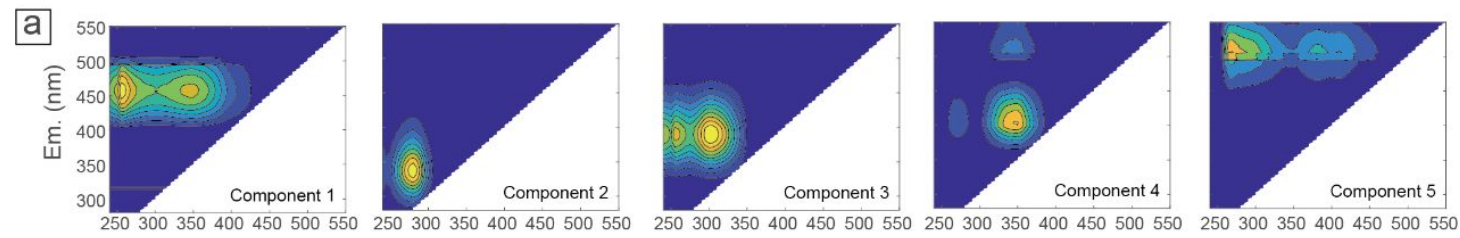
Ex. $(\mathrm{nm})$

b
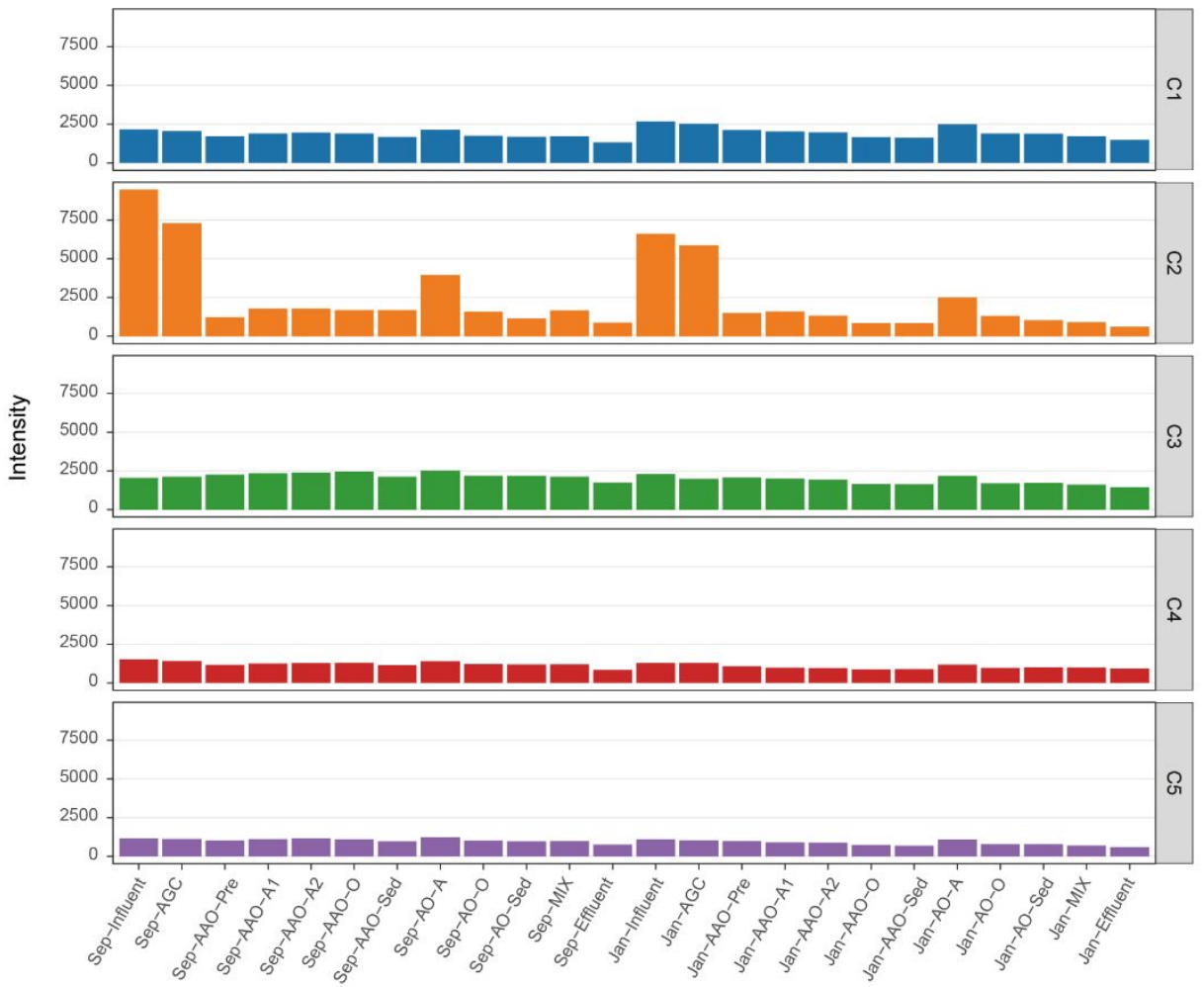

Figure S2 Changes of PARAFAC-identified DOM components' intensities. (a) Ex and Em of components. (b) Intensities of components. 

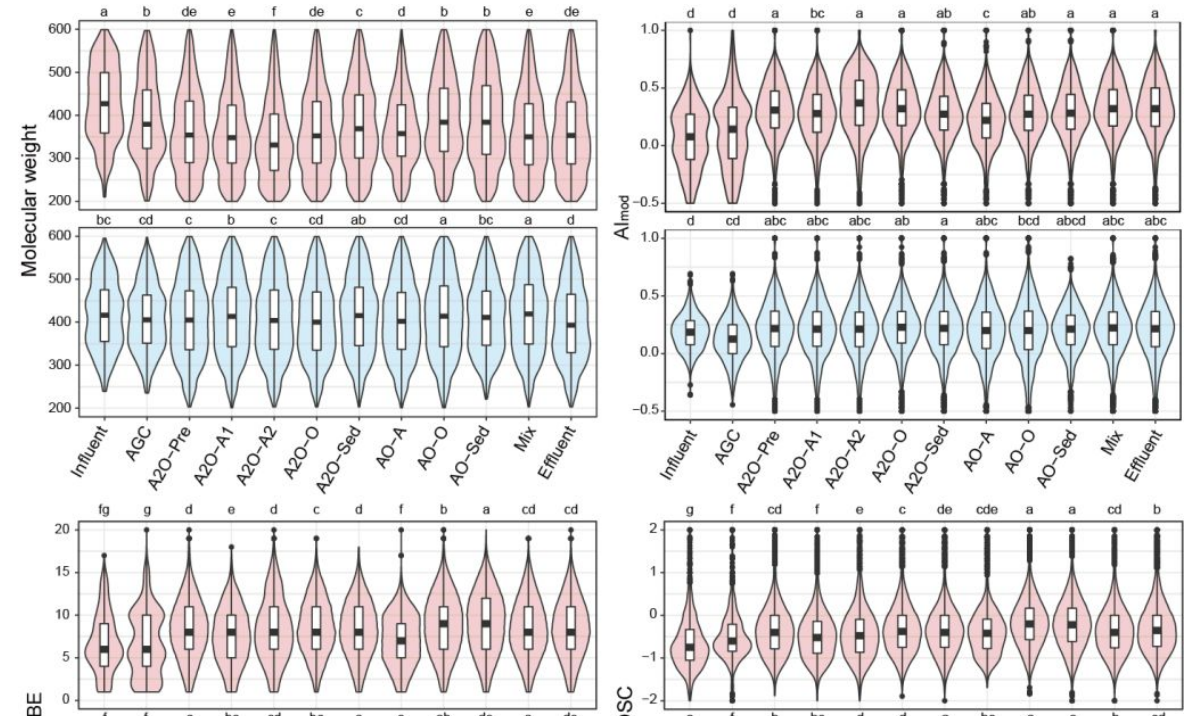

ㅁำ
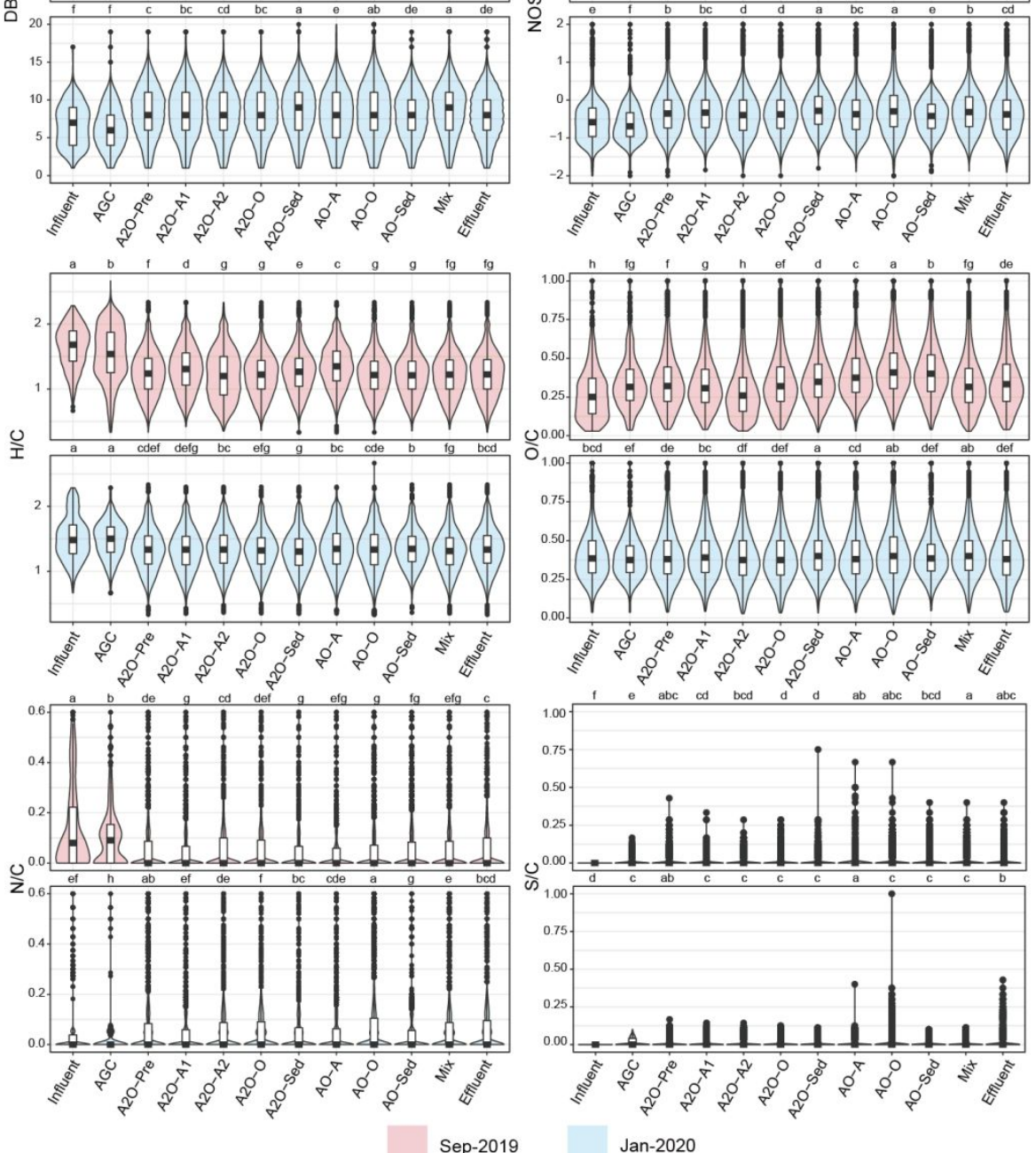
Figure S3 Distributions of DOM molecules' chemical attributes. MW, molecular weight. DBE, double bond equivalence. Letters indicated the significance test of Tukey HSD test at $a=0.05$. The difference between paired groups with identical letter is insignificant. 

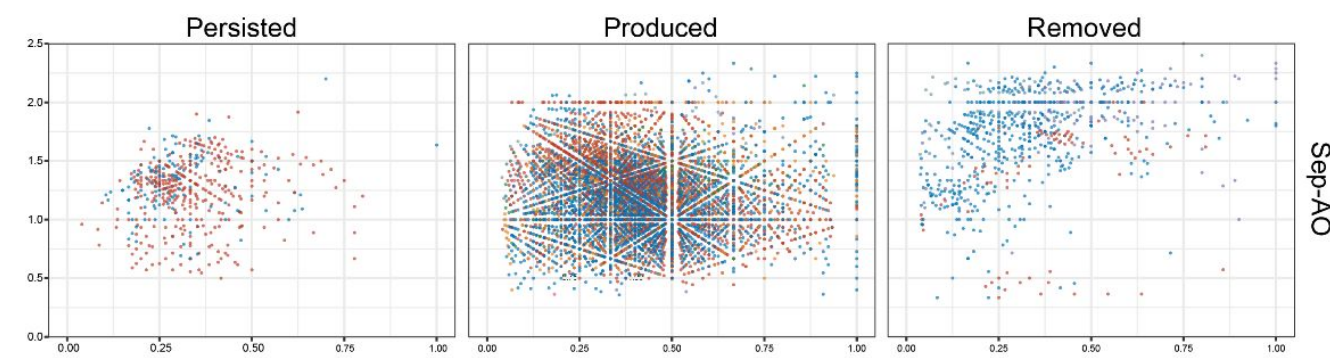

0
$\mathbb{D}$
0
1
0
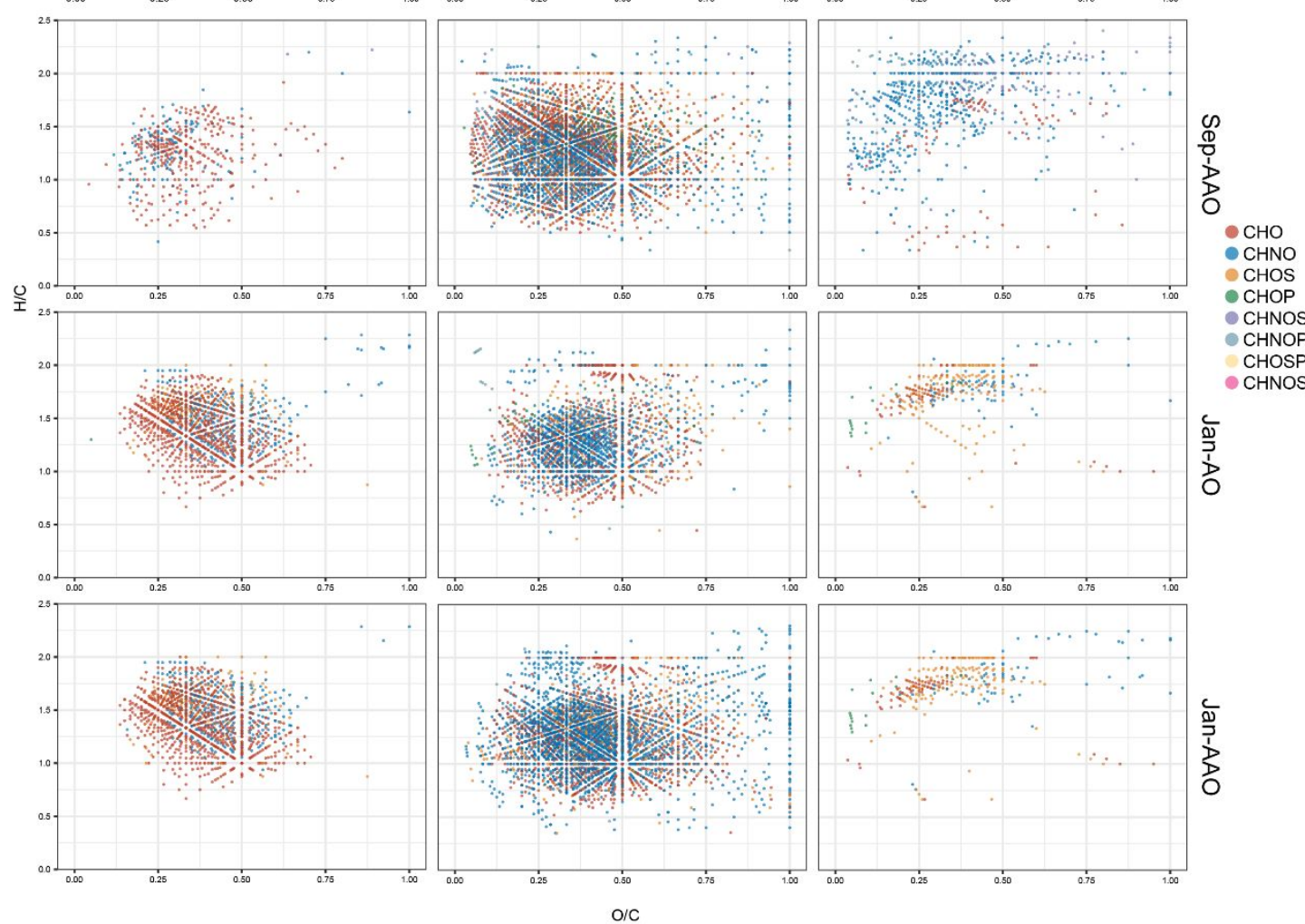

变

Figure S4 Separated van Krevelen diagrams of the produced, persisted, and removed

DOM molecules in biotreatment processes. Removed means they are in the AGC but become undetected after biotreatments. Persisted means they are present in the AGC and also after biotreatments. Produced means that they are not in the AGC but present after biotreatments. 

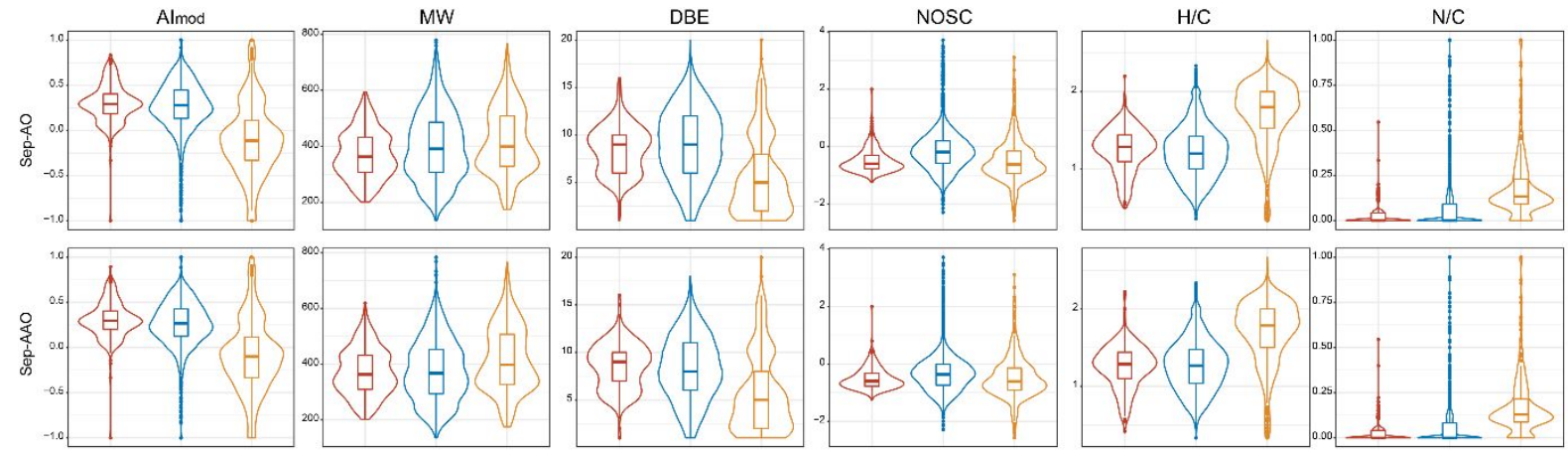

EPersisted Eroduced
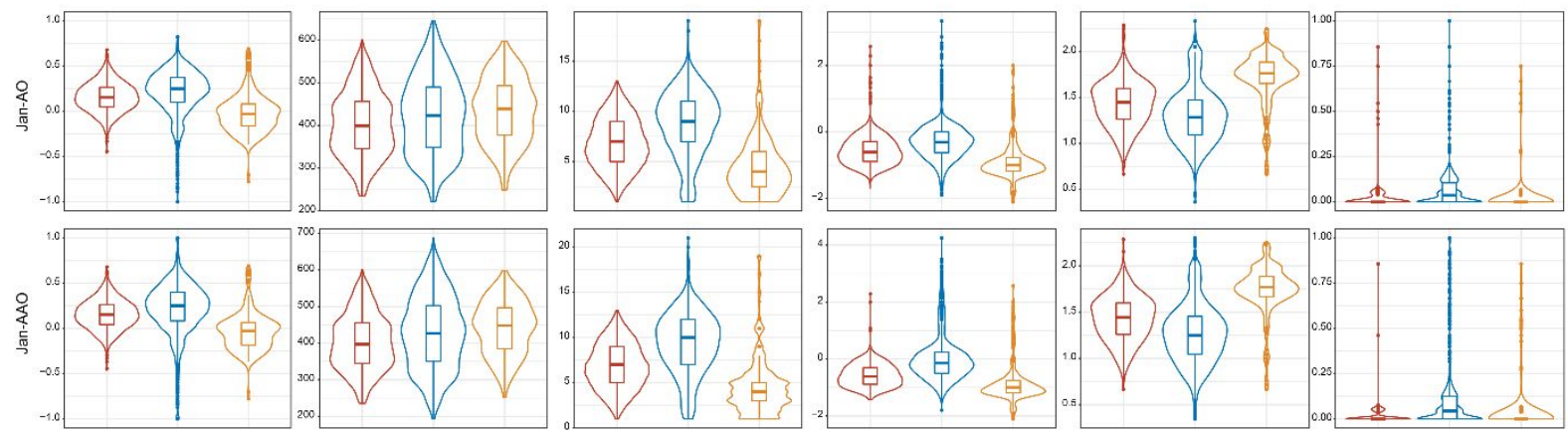

Figure S5 Distributions of removed/persisted/produced DOM molecules' chemical

attributes. Metagenome-assembled genomes (MAGs) with abundance $<1$ CoPM were omitted from the graph. Significance is determined by Student's t-test. 


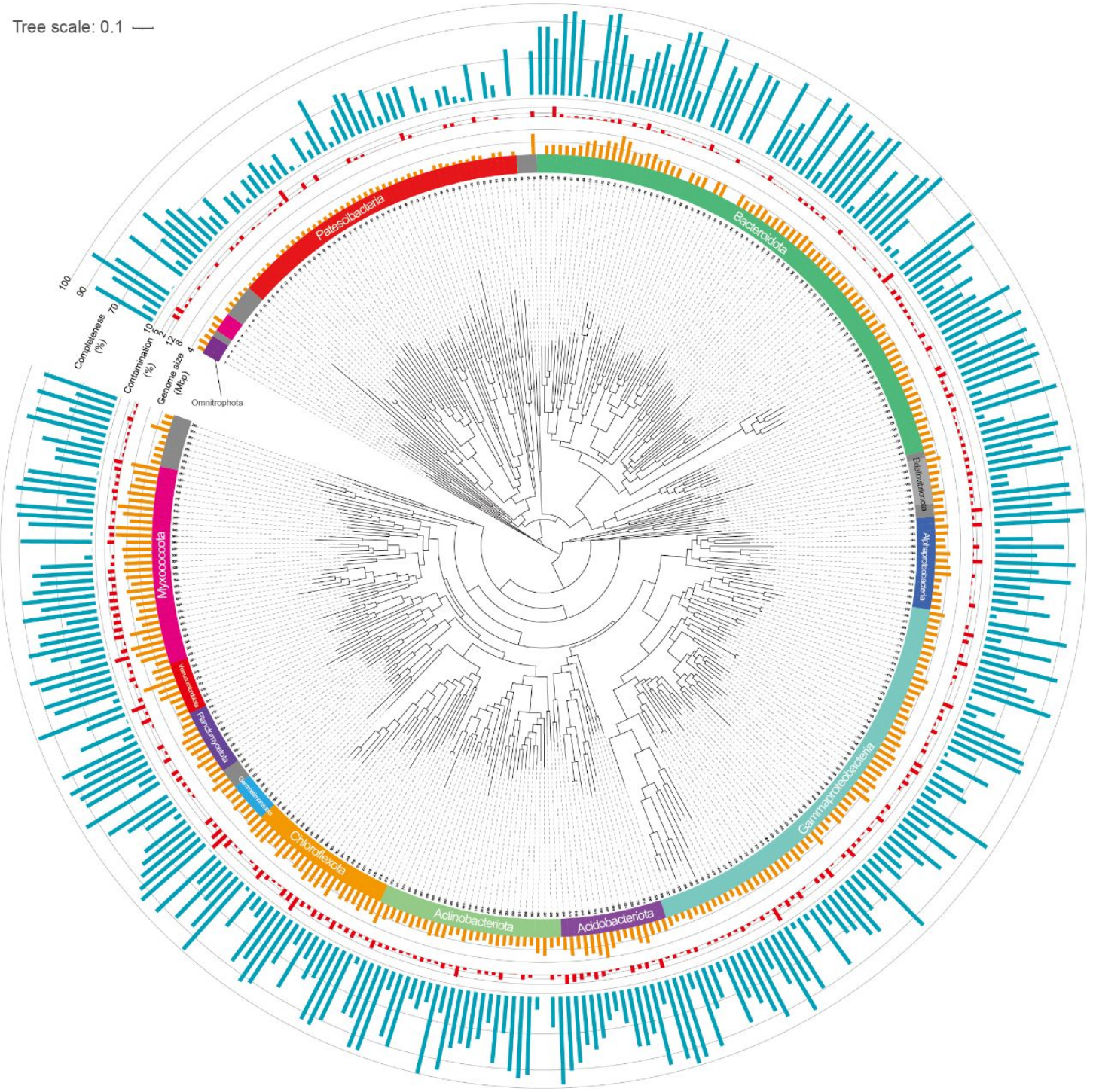

Figure S6 The phylogenetic tree of microbes inhabiting $\mathrm{A} / \mathrm{A} / \mathrm{O}$ and $\mathrm{A} / \mathrm{O}$ processes. The rounded strips in different colors indicate the phylum-level taxonomy of MAGs. The bar plot from inner to outer indicates genome size (Mbp), contamination (\%), and completeness (\%).

The genome size is estimated as actual assembly genome size/estimated coverage/ $(1+$ estimated contamination). 


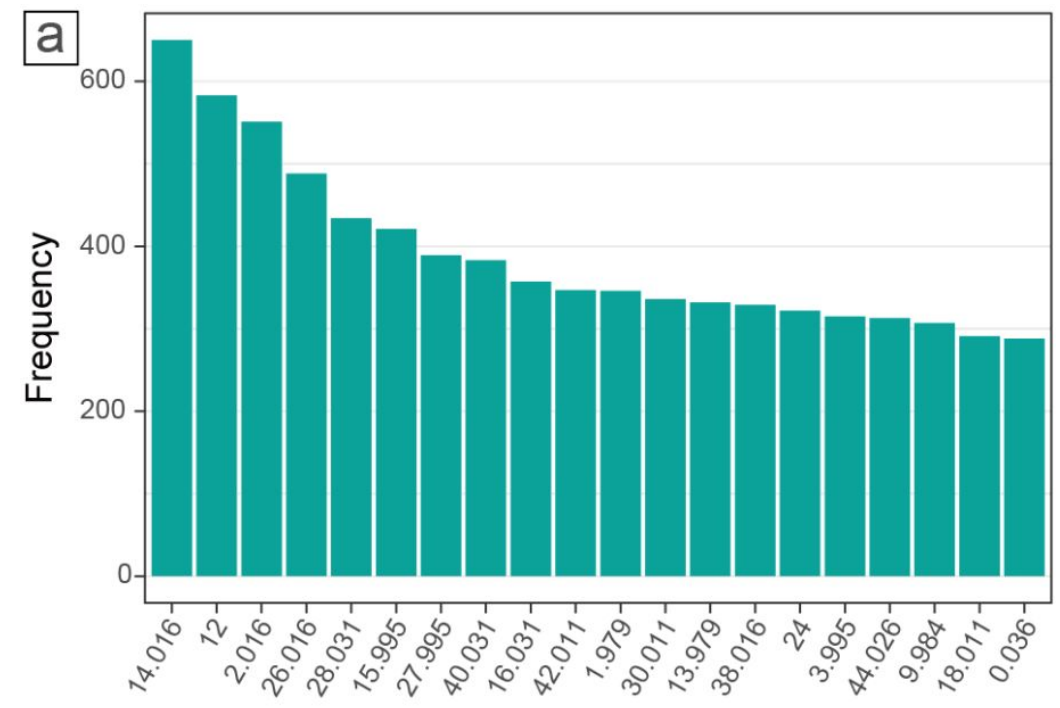

Top 20 PMDs

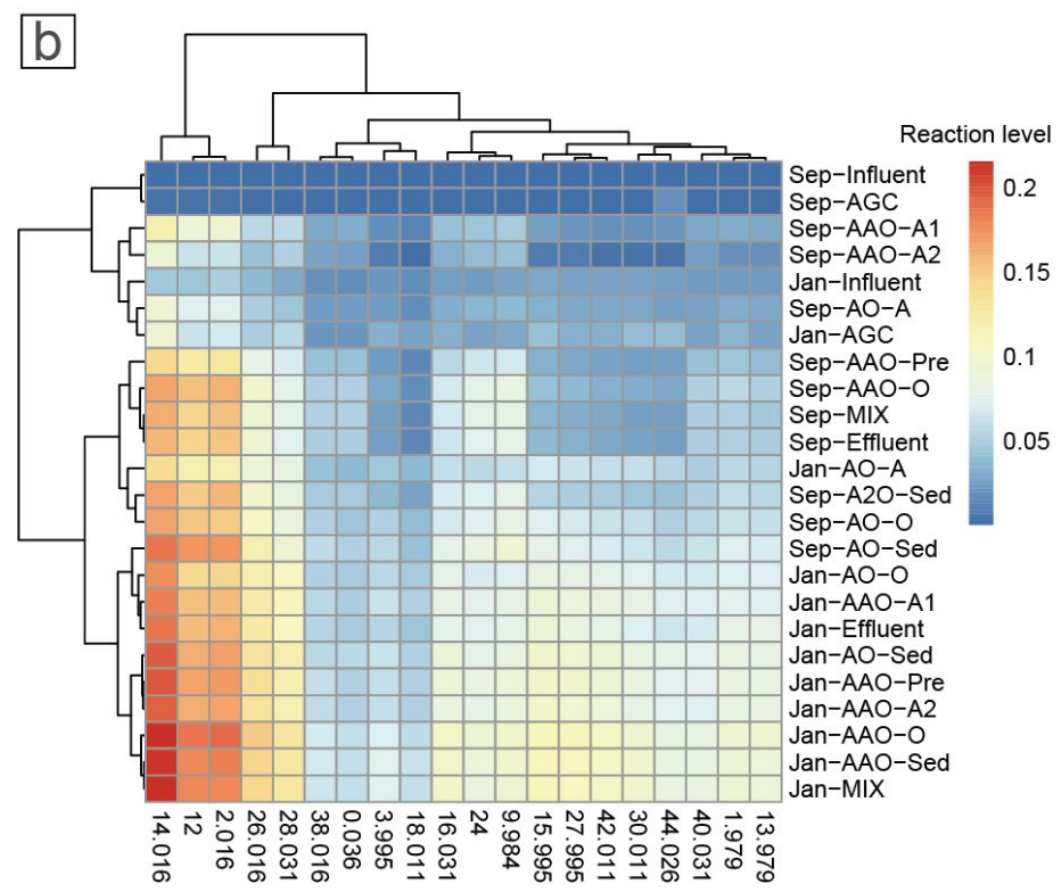

Figure S7 Frequencies and abundances of individual PMDs. (a) Frequencies of PMDs in the co-occurrence network. (b) Abundance (reaction level) of PMD. 
a

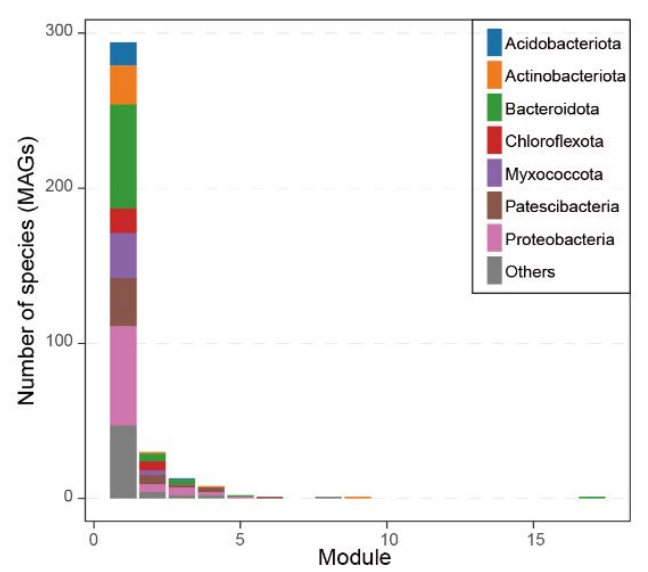

C b

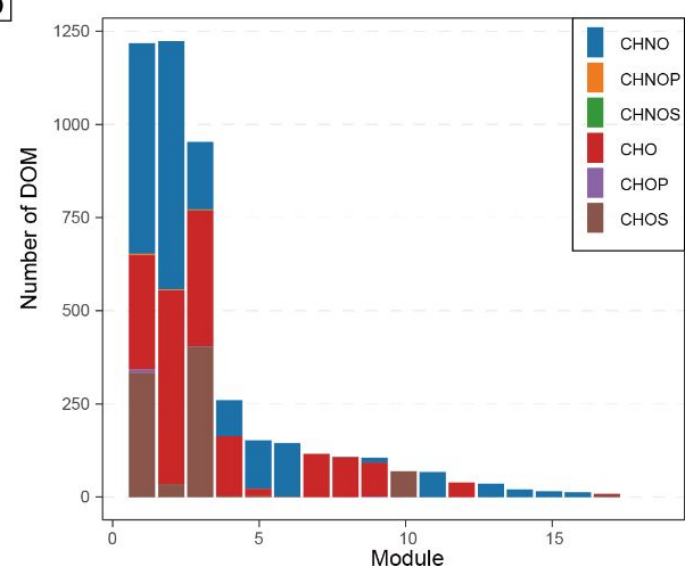

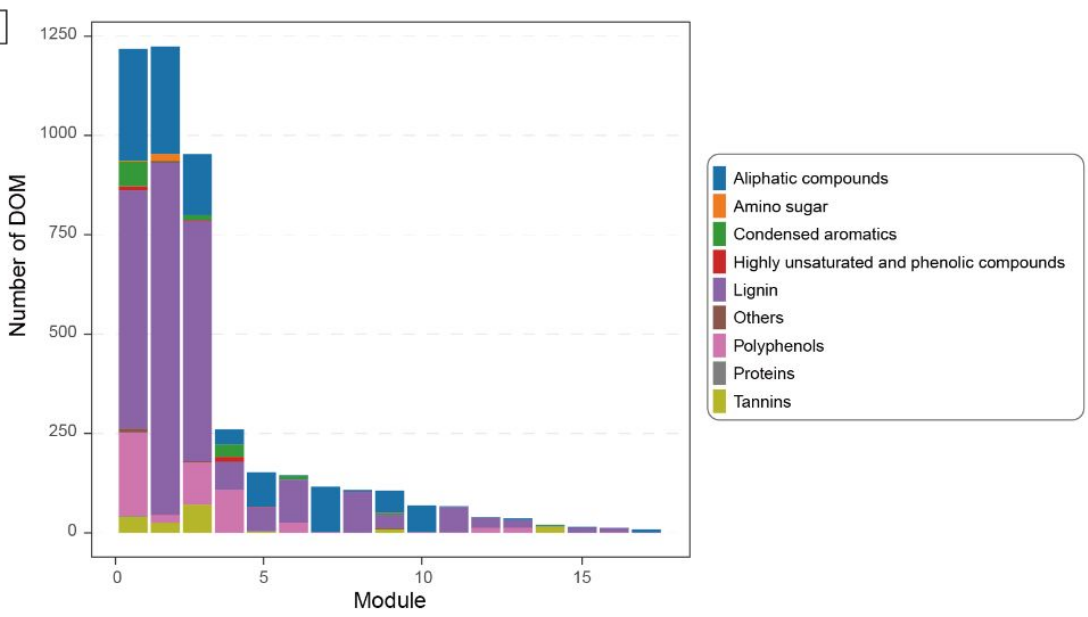

Figure S8 Module-wise distribution of MAGs and DOM molecules. (a) Distribution of microbes at phylum-level. (b) Distribution of DOM in group categories. (c) Distribution of DOM in compound categories. 


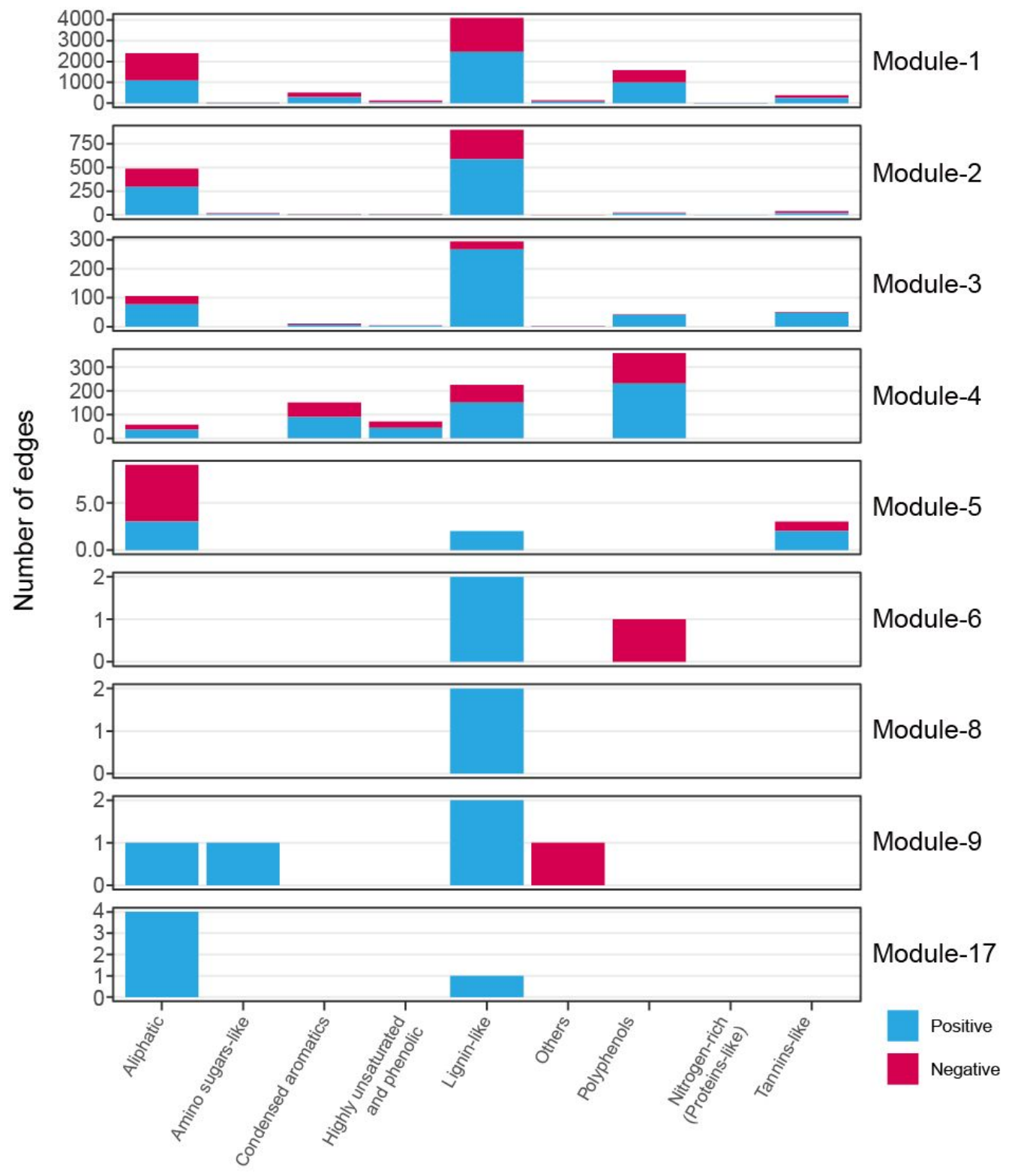

Figure S9 Correlation type (positive or negative) between MAGs and DOM molecules within modules. 


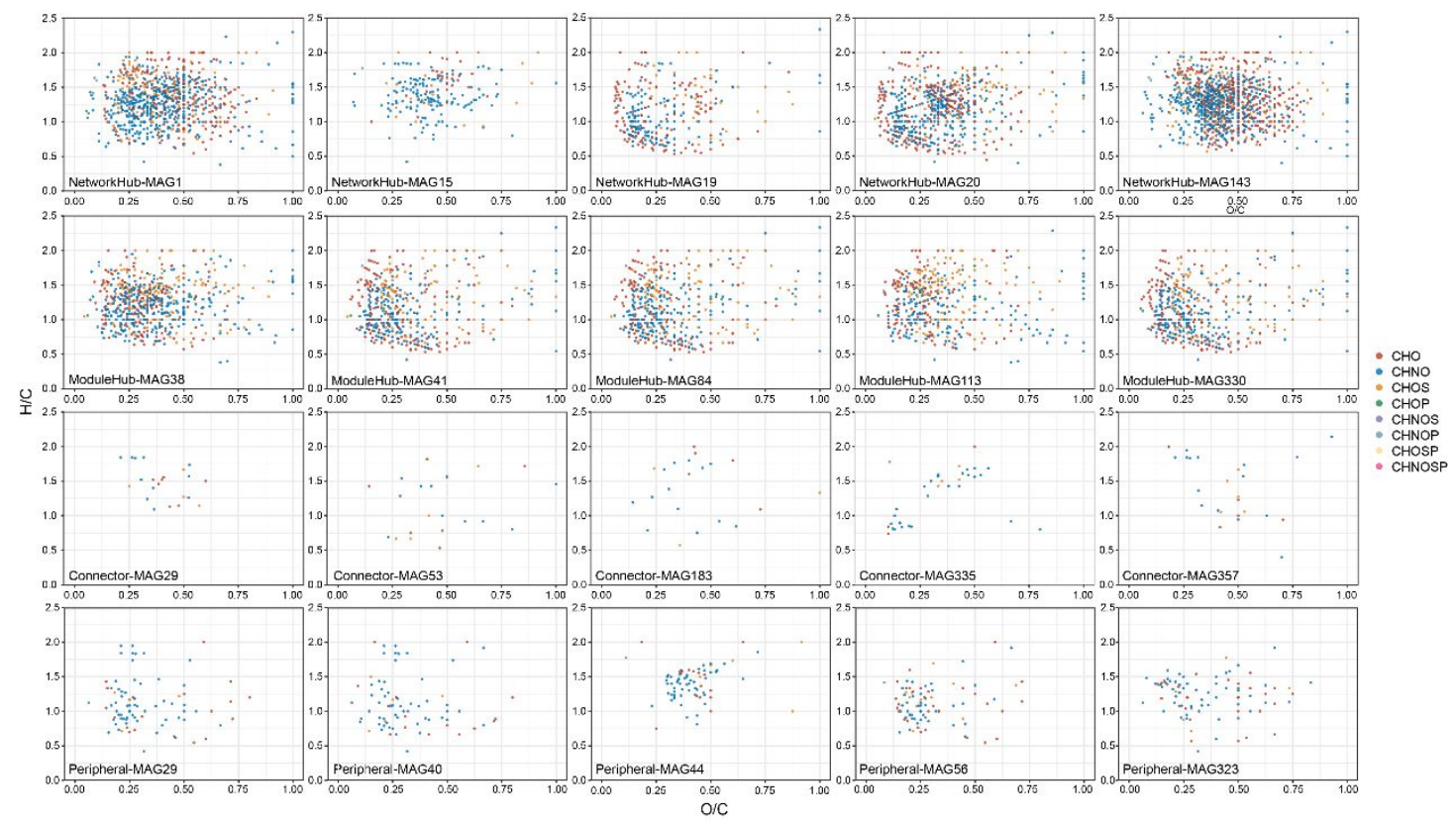

Figure S10 DOM molecules associated with representative network hubs, module hubs, connectors, and peripherals. Representative microbes are the top five MAGs in each role in term of degree. 

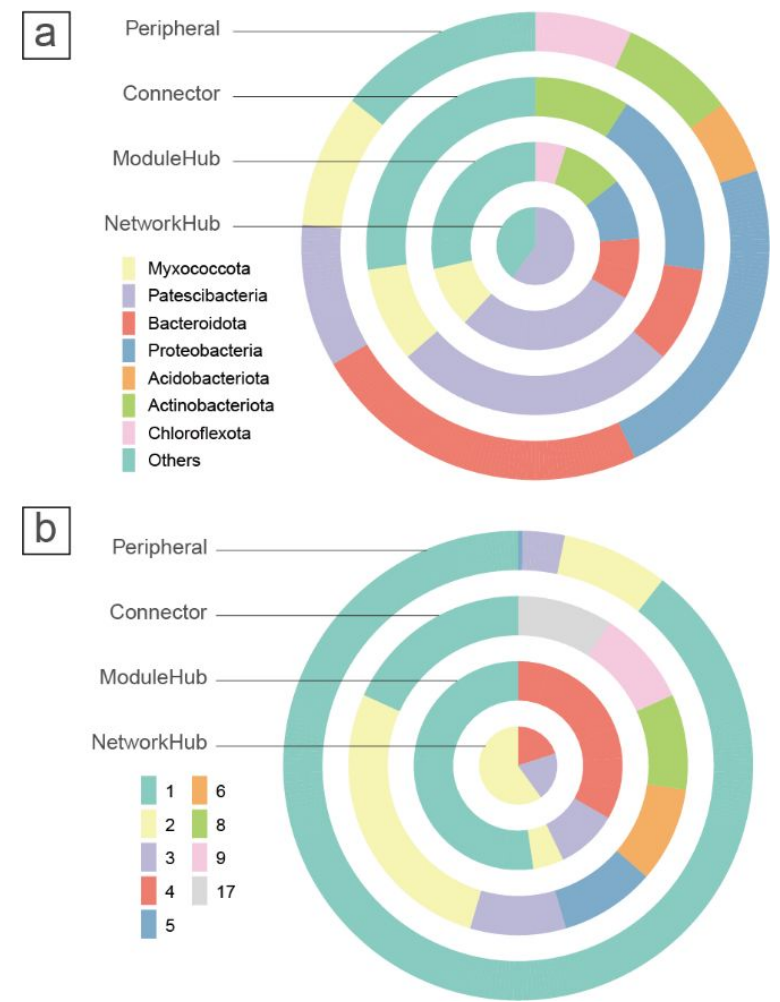

Figure S11 Distribution of microbial network hubs, module hubs, connectors, and peripherals in modules. (a) Taxonomic distribution of different roles. (b) Module distribution of different roles. 


\section{Supplementary References}

1. Stedmon, C. A.; Bro, R., Characterizing dissolved organic matter fluorescence with parallel factor analysis: a tutorial. Limnology and Oceanography: Methods 2008, 6, (11), 572579.

2. Zheng, Q.; Chen, Q.; Cai, R.; He, C.; Guo, W.; Wang, Y.; Shi, Q.; Chen, C.; Jiao, N., Molecular characteristics of microbially mediated transformations of Synechococcus-derived dissolved organic matter as revealed by incubation experiments. Environ. Microbiol. 2019, 21, (7), 2533-2543.

3. Zhang, B.; Shan, C.; Hao, Z.; Liu, J.; Wu, B.; Pan, B., Transformation of dissolved organic matter during full-scale treatment of integrated chemical wastewater: Molecular composition correlated with spectral indexes and acute toxicity. Water Res. 2019, 157, 472482.

4. Kellerman, A. M.; Dittmar, T.; Kothawala, D. N.; Tranvik, L. J., Chemodiversity of dissolved organic matter in lakes driven by climate and hydrology. Nat. Commun. 2014, 5, 8. 5. D'Andrilli, J.; Cooper, W. T.; Foreman, C. M.; Marshall, A. G., An ultrahigh-resolution mass spectrometry index to estimate natural organic matter lability. Rapid Commun Mass Spectrom. 2015, 29, (24), 2385-2401.

6. Gotelli, N. J.; Chao, A., Measuring and Estimating Species Richness, Species Diversity, and Biotic Similarity from Sampling Data. In Encyclopedia of Biodiversity (Second Edition), Levin, S. A., Ed. Academic Press: Waltham, 2013; pp 195-211.

7. Mentges, A.; Feenders, C.; Seibt, M.; Blasius, B.; Dittmar, T., Functional Molecular Diversity of Marine Dissolved Organic Matter Is Reduced during Degradation. Frontiers in Marine Science 2017, 4, 10.

8. Maizel, A. C.; Remucal, C. K., The effect of advanced secondary municipal wastewater treatment on the molecular composition of dissolved organic matter. Water Res. 2017, 122, $42-52$.

9. Zhou, L.; Zhou, Y.; Tang, X.; Zhang, Y.; Jang, K.-S.; Székely, A. J.; Jeppesen, E., Resource aromaticity affects bacterial community successions in response to different sources of dissolved organic matter. Water Res. 2021, 190, 116776.

10. Peng, X.; Wilken, S. E.; Lankiewicz, T. S.; Gilmore, S. P.; Brown, J. L.; Henske, J. K.; Swift, C. L.; Salamov, A.; Barry, K.; Grigoriev, I. V.; Theodorou, M. K.; Valentine, D. L.; O'Malley, M. A., Genomic and functional analyses of fungal and bacterial consortia that enable lignocellulose breakdown in goat gut microbiomes. Nature Microbiology 2021.

11. Bolger, A. M.; Lohse, M.; Usadel, B., Trimmomatic: a flexible trimmer for Illumina sequence data. Bioinformatics 2014, 30, (15), 2114-2120.

12. Li, D.; Liu, C.-M.; Luo, R.; Sadakane, K.; Lam, T.-W., MEGAHIT: an ultra-fast singlenode solution for large and complex metagenomics assembly via succinct de Bruijn graph. Bioinformatics 2015, 31, (10), 1674-1676.

13. Wu, Y.-W.; Tang, Y.-H.; Tringe, S. G.; Simmons, B. A.; Singer, S. W., MaxBin: an automated binning method to recover individual genomes from metagenomes using an expectation-maximization algorithm. Microbiome 2014, 2, (1), 26.

14. Kang, D. D.; Li, F.; Kirton, E.; Thomas, A.; Egan, R.; An, H.; Wang, Z., MetaBAT 2: an adaptive binning algorithm for robust and efficient genome reconstruction from metagenome assemblies. PeerJ 2019, 7, e7359.

15. Alneberg, J.; Bjarnason, B. S.; de Bruijn, I.; Schirmer, M.; Quick, J.; Ijaz, U. Z.; Lahti, L.; Loman, N. J.; Andersson, A. F.; Quince, C., Binning metagenomic contigs by coverage and composition. Nat. Methods 2014, 11, 1144.

16. Uritskiy, G. V.; DiRuggiero, J.; Taylor, J. J. M., MetaWRAP—a flexible pipeline for genome-resolved metagenomic data analysis. Microbiome 2018, 6, (1), 158.

17. Parks, D. H.; Rinke, C.; Chuvochina, M.; Chaumeil, P.-A.; Woodcroft, B. J.; Evans, P. 
N.; Hugenholtz, P.; Tyson, G. W., Recovery of nearly 8,000 metagenome-assembled genomes substantially expands the tree of life. Nature Microbiology 2017, 2, (11), 1533-1542.

18. Parks, D. H.; Imelfort, M.; Skennerton, C. T.; Hugenholtz, P.; Tyson, G. W., CheckM: assessing the quality of microbial genomes recovered from isolates, single cells, and metagenomes. Genome Res. 2015, 25, (7), 1043-1055.

19. Lowe, T. M.; Eddy, S. R., tRNAscan-SE: A Program for Improved Detection of Transfer RNA Genes in Genomic Sequence. Nucleic Acids Res. 1997, 25, (5), 955-964.

20. Nawrocki, E. P.; Kolbe, D. L.; Eddy, S. R., Infernal 1.0: inference of RNA alignments. Bioinformatics 2009, 25, (10), 1335-1337.

21. Bowers, R. M.; Kyrpides, N. C.; Stepanauskas, R.; Harmon-Smith, M.; Doud, D.; Reddy, T. B. K.; Schulz, F.; Jarett, J.; Rivers, A. R.; Eloe-Fadrosh, E. A.; Tringe, S. G.; Ivanova, N. N.; Copeland, A.; Clum, A.; Becraft, E. D.; Malmstrom, R. R.; Birren, B.; Podar, M.; Bork, P.; Weinstock, G. M.; Garrity, G. M.; Dodsworth, J. A.; Yooseph, S.; Sutton, G.; Glöckner, F. O.; Gilbert, J. A.; Nelson, W. C.; Hallam, S. J.; Jungbluth, S. P.; Ettema, T. J. G.; Tighe, S.; Konstantinidis, K. T.; Liu, W.-T.; Baker, B. J.; Rattei, T.; Eisen, J. A.; Hedlund, B.; McMahon, K. D.; Fierer, N.; Knight, R.; Finn, R.; Cochrane, G.; Karsch-Mizrachi, I.; Tyson, G. W.; Rinke, C.; Kyrpides, N. C.; Schriml, L.; Garrity, G. M.; Hugenholtz, P.; Sutton, G.; Yilmaz, P.; Meyer, F.; Glöckner, F. O.; Gilbert, J. A.; Knight, R.; Finn, R.; Cochrane, G.; Karsch-Mizrachi, I.; Lapidus, A.; Meyer, F.; Yilmaz, P.; Parks, D. H.; Murat Eren, A.; Schriml, L.; Banfield, J. F.; Hugenholtz, P.; Woyke, T.; The Genome Standards, C., Minimum information about a single amplified genome (MISAG) and a metagenomeassembled genome (MIMAG) of bacteria and archaea. Nat. Biotechnol. 2017, 35, (8), 725 731.

22. Chaumeil, P.-A.; Mussig, A. J.; Hugenholtz, P.; Parks, D. H., GTDB-Tk: a toolkit to classify genomes with the Genome Taxonomy Database. Bioinformatics 2019, 36, (6), 19251927.

23. He, C.; Keren, R.; Whittaker, M. L.; Farag, I. F.; Doudna, J. A.; Cate, J. H. D.; Banfield, J. F., Genome-resolved metagenomics reveals site-specific diversity of episymbiotic CPR bacteria and DPANN archaea in groundwater ecosystems. Nature Microbiology 2021. 24. Zhou, Z.; Tran, P.; Liu, Y.; Kieft, K.; Anantharaman, K., METABOLIC: A scalable high-throughput metabolic and biogeochemical functional trait profiler based on microbial genomes. 2019, 761643. 